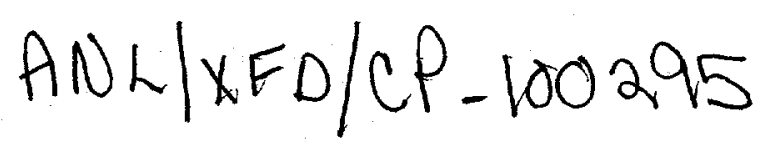

\title{
An Electromagnetic Helical Undulator for
Polarized X-Rays ${ }^{*}$
} Evtushenko $^{2}$, V. Kolmogorov ${ }^{2}$, P. Vobly ${ }^{2}$, E. Antokhin ${ }^{2}$, P. Ivanov ${ }^{2}$, I. B. Vasserman ${ }^{1}$, E. M. Trakhtenberg ${ }^{1}$, P.K. Den Hartog ${ }^{1}$, B. Deriy ${ }^{1}$, M. Erdmann ${ }^{1}$, O. Makarov ${ }^{1}$, E. R. Moog $^{1}$

\author{
${ }^{1}$ Advanced Photon Source, Argonne National Laboratory, Argonne IL 60439 \\ ${ }^{2}$ Budker Institute of Nuclear Physics, 11 Ac. Lavrentyev Prosp. 630090 Novosibirsk, Russia
}

\begin{abstract}
Linearly and circularly polarized $x$-rays have been very successfully applied to the study of the properties of materials. Many applications can benefit from the availability of energy-tunable, high-brilliance $\mathrm{x}$-ray beams with adjustable polarization properties. A helical undulator that can generate beams of variable (linear to circular) polarization has been designed and built by the Budker Institute of Nuclear Physics and the Advanced Photon Source. The first harmonic of this $12.8-\mathrm{cm}$-period device will cover the energy range from $0.4 \mathrm{keV}$ to $3.5 \mathrm{keV}$. An important feature of this fully electromagnetic device is that it will allow us to generate $100 \%$ horizontally $\left(\mathrm{K}_{\mathrm{x}}=0\right)$ or vertically $\left(\mathrm{K}_{\mathrm{y}}=0\right)$ plane-polarized radiation, which will enable many experiments otherwise not technically feasible. With symmetric deflection parameters $\left(\mathrm{K}_{\mathrm{x}}=\mathrm{K}_{\mathrm{y}}\right)$, the on-axis radiation will be circularly polarized, with a user-selectable handedness. The polarization can be changed at rates up to $10 \mathrm{~Hz}$.
\end{abstract}

\section{INTRODUCTION}

Studies of the magnetic properties of materials have been limited by the lack of energy-tunable, high-brilliance $\mathrm{x}$-ray beams with adjustable polarization properties. Op-tics can be fabricated to simultaneously provide a high-quality beam and adjust-able polarization using the standard Advanced Photon Source (APS) undulator A as a source, but only in the energy range above $5 \mathrm{keV}$. To extend the range below 5 $\mathrm{keV}$, the Budker Institute and the APS have collaborated to build a fully electromagnetic helical undulator that can generate light of variable (linear to circular) polarization in this lower energy regime. The polarization can be linear vertical, linear horizontal, or elliptical to circular of either handedness. The expected brilliance of the CPU operating in circular and linear modes is shown in Fig. 1. The curves are calculated for an ideal magnetic field and for: $7.0 \mathrm{GeV}, 100 \mathrm{~mA}, \sigma_{x}=0.359 \mathrm{~mm}$, $\sigma_{y}=0.021 \mathrm{~mm}, \sigma_{x^{\prime}}=0.0230 \mathrm{mrad}, \sigma_{y^{\prime}}=0.0039 \mathrm{mrad}$, and a beam energy spread of $0.1 \%$. The parameters for the device are shown in Table I.

*Supported by the U.S. Dept. of Energy, BES-Materials Sciences, under Contract No. W-31-109Eng-38

The submitted manuscript has been created by the University of Chicago as Operator of Argonne National Laboratory ("Argonne") under Contract No. W-31-109-ENG-38 with the U.S. Department of Energy. The U.S. Government retains for itself, and others acting on its behalf, a paid-up, nonexclusive, irrevocable worldwide license in said article to reproduce, prepare derivative works, distribute copies to the public, and perform publicly and display publicly, by or on behalf of the Government. 


\section{DISCLAIMER}

This report was prepared as an account of work sponsored by an agency of the United States Government. Neither the United States Government nor any agency thereof, nor any of their employees, make any warranty, express or implied, or assumes any legal liability or responsibility for the accuracy, completeness, or usefulness of any information, apparatus, product, or process disclosed, or represents that its use would not infringe privately owned rights. Reference herein to any specific commercial product, process, or service by trade name, trademark, manufacturer, or otherwise does not necessarily constitute or imply its endorsement, recommendation, or favoring by the United States Government or any agency thereof. The views and opinions of authors expressed herein do not necessarily state or reflect those of the United States Government or any agency thereof. 


\section{DISCLAIMER}

Portions of this document may be illegible in electronic image products. Images are produced from the best available original document. 


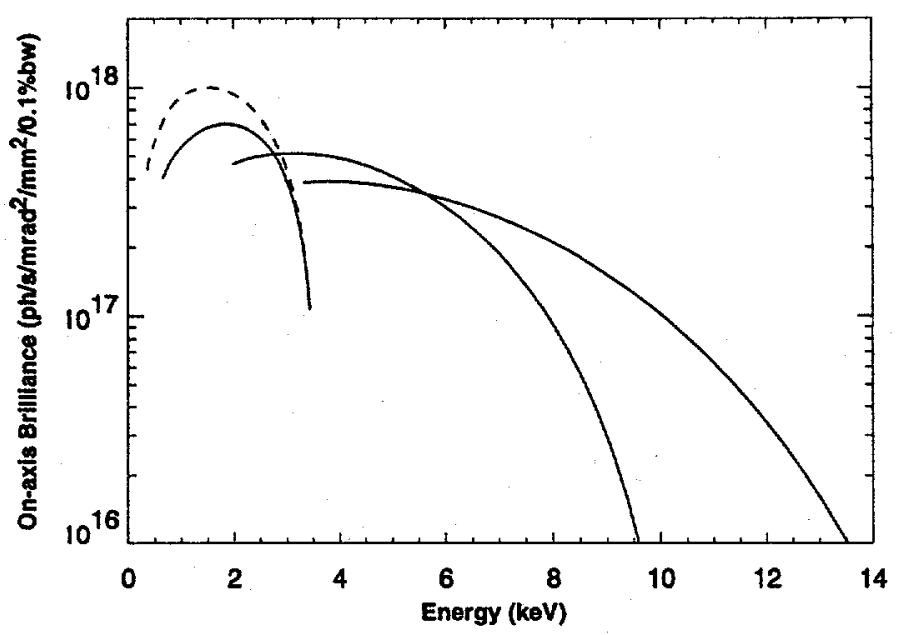

FIGURE 1. The brilliance range of the CPU is shown for circular (dashed) and linear (solid) polarization modes. The first three odd harmonics are shown for the linear mode.

\section{MAGNETIC DESIGN}

The 12.8-cm-period device uses electromagnets for both horizontal and vertical fields. The CPU will share a straight section with a standard planar undulator, so the CPU is made compatible with the APS standard insertion device vacuum chamber (1). This means that the ID is constructed in a $\mathrm{C}$ geometry with poles above and below the vacuum chamber, so that one side is left open for installation on the ring. The vacuum chamber can be installed on the storage ring before ID installation, and the CPU can be installed or removed without venting any portion of the vacuum system. The horizontal field is produced by appropriately powering coils on four poles that are displaced both transversely and vertically with respect to the beam (see figure 2) and are

TABLE 1. CPU Design Parameters

\begin{tabular}{lll}
\hline Parameter Description & Value & Units \\
\hline Units & & \\
Period & 12.8 & $\mathrm{~cm}$ \\
Number of horizontal poles & 34 & \\
Number of vertical poles & 34 & \\
Vertical or horizontal full-field poles & 28 & $\mathrm{~m}$ \\
Overall length & 2.3 & $\mathrm{~mm}$ \\
Vertical pole gap & 11 & Tesla \\
Maximum magnetic field & 0.24 & Tesla \\
Horizontal peak field at 1400 amps & 0.287 & Tesla \\
Vertical peak field at 350 amps & 0.31 & $\mathrm{Amp}$ \\
Current to produce 0.28 T vertically & 315 & $\mathrm{Amp}$ \\
Current to produce 0.28 T horizontally & 1350 & $\mathrm{~Hz}$ \\
Switching frequency & $0-10$ & $\mathrm{Ms}$ \\
Switching rise time (including overshoot) & $<20$ & $\%$ \\
Electromagnet DC stability & $<1$ & $\mathrm{~W}$ \\
Maximum total radiation power & 800 & $\mathrm{~W} / \mathrm{mm}^{2}$ \\
Maximum radiation power density & 17 & \\
\hline
\end{tabular}




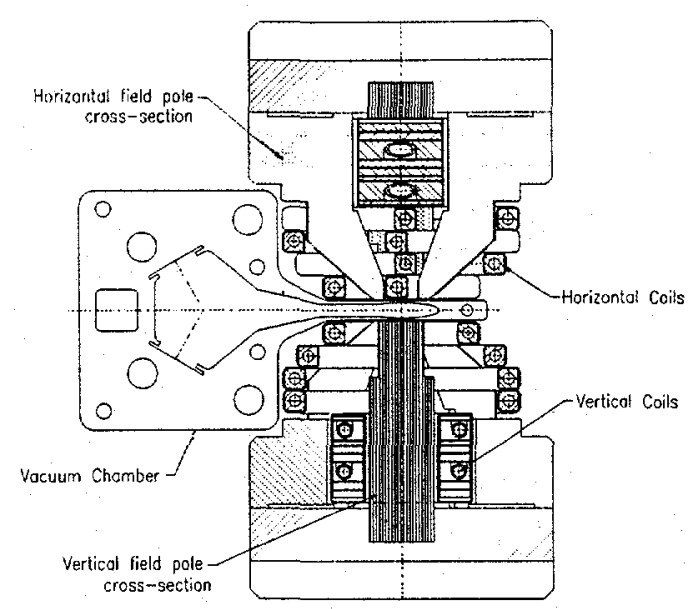

FIGURE 2. Cross section of the CPU poles nested around the ID vacuum chamber. The horizontal pole cross section is shown above the chamber; the vertical pole cross section below.

displaced longitudinally from the vertical poles by one-quarter period. The coils that produce the horizontal field snake their way among the poles in four layers so as to appropriately polarize the horizontal field poles while, ideally, not producing any vertical field component. Separate coils power the vertical field poles.

\section{MAGNETIC FIELD COMPENSATION}

There are 34 poles each for the horizontal and vertical fields, including the ends. At both ends of the device and in both field directions, the field strength pattern for the end poles is $0,1 / 4,3 / 4,1, \ldots$ This pattern was chosen to minimize steering and displacement at the ends. The 'zero-strength' horizontal and vertical end poles at the upstream and downstream ends have separately powered compensation coils, so the first and second field integrals through the undulator can be adjusted independently. Arbitrary function generators will be used to adjust the currents in the coils so that a transient magnetic field due to changing or reversing the current in the coils will also be canceled.

Requirements are also imposed on the integrated multipole moments of the field. Although there is certainly a dependence of the field strength with transverse position even in a perfect undulator (the 'roll-off'), the multipole moments of the integrated field through this perfect undulator (with an integer number of periods) should be small. Integrated multipole moments arise from imperfections in the magnetic materials and from finite engineering tolerances. The CPU has integrated multipole moments that are larger than our requirements. Even at zero current, there is a residual integrated moment due to a remanent magnetization. Initial attempts to demagnetize were unsuccessful, and we are proceeding with other correction methods. A coil is being added to the zero-strength horizontal field poles at the end of the undulator, where they are the last poles. The direction of the current in the windings is set to produce a quadrupole field instead of a horizontal field. These windings will be used to cancel the integrated quadrupole field. We plan to place an additional electromag- 
net at the end of the undulator, to correct the integrated skew quadrupole moment. This additional magnet will have poles directly above and below the beam, with the same polarity, but will have no poles in the transverse direction.

Although ideally there should be no cross-talk between the vertical and horizontal field components, in reality we find that there is. One origin of possible cross-talk is a changing permeability in the poles-for example, the horizontal flux that is shunted through the vertical field poles will change when the vertical field changes because the vertical flux in those poles changes. Another source of cross-talk is the imperfect shaping and placement of the snakelike horizontal field coils and their proximity to the undulator gap. Cross-talk between the vertical and horizontal fields does not necessarily need to be corrected, but coil currents do need to be calibrated in order to produce radiation with the desired polarization and the desired energy.

The overall effect of the current-dependent integrated multipole moments, the multipole moments resulting from the remanent field, and the effect of the cross-talk on the moments is that there will be a complicated set of multipole corrections. The currents needed in the multipole correction coils will be determined over the full range of settings for the vertical and horizontal currents.

\section{UNDULATOR POWER SUPPLY}

The CPU power supply (PS) is designed to supply the undulator coils with DC, AC or pulsed current. It has 8 channels: one each for horizontal and vertical coils and six channels to supply the correcting coils. The PS produces an output current up to $1600 \mathrm{~A}$ (voltage up to $20 \mathrm{~V}$ ) for the horizontal coil and up to $400 \mathrm{~A}$ (voltage up to $10 \mathrm{~V}$ ) for the vertical coil. Channels supplying the correcting coils can provide up to $+/-5$ A with voltages up to $10 \mathrm{~V}$. Both main channels can supply the load with positive or negative DC, AC current with a trapezoidal shape and a tunable repetition frequency, or pulsed current of positive or negative polarities. Forced switching of the current polarity is accomplished in a few milliseconds, seven times faster than the normal transient process. As a result, operation at a frequency of up to $10 \mathrm{~Hz}$ is possible.

\section{SUMMARY}

A new type of undulator for polarized radiation has been built and is undergoing final tuning at the APS. We plan to complete the tuning in late 1999 and to install the undulator in the storage ring in early 2000 .

\section{REFERENCE}

Den Hartog, P., Grimmer, J., Trakhtenberg, E., Wiemerslage, G., Xu, S., "Advanced Photon Source Experience with Vacuum Chambers for Insertion Devices," Proc. PAC '97, Vancouver, B.C., May 1997. 